\title{
VA Provider Perspectives on Coordinating COPD Care Across Health Systems
}

\author{
Seppo T. Rinne, MD PhD, Kirsten Resnick, MS, Renda Soylemez Wiener, MD MPH, \\ Steven R. Simon, MD MPH, and A. Rani Elwy, PhD
}

Bedford, MA, USA.

BACKGROUND: More and more Veterans are receiving care from community providers, increasing the need for effective coordination across health systems. For Veterans with chronic obstructive pulmonary disease (COPD), this need is intensified by complex comorbidity patterns that often include multiple providers co-managing patient care. OBJECTIVES: We sought to understand how VA providers perceive coordination with community providers for Veterans with COPD.

DESIGN: Qualitative study of VA providers.

METHODS: We selected six geographically diverse VA sites and conducted semi-structured telephone interviews with providers practicing in inpatient and/or outpatient settings who care for Veterans with COPD.

MAIN MEASURES: Interviews focused on communication with community providers about discharge information and clinic management. We analyzed responses according to the principles of conventional content analysis, allowing inductive themes to emerge.

KEY RESULTS: We interviewed 25 providers during the period of June to October 2017. Qualitative data analysis yielded five themes: (1) VA providers perceive communication challenges between VA and community providers, including difficult, inadequate, and delayed communication; (2) communication is facilitated by personal relationships across health systems; (3) the lack of electronic health record (EHR) interoperability impairs communication, resulting in transmission of unstructured data; (4) poor communication leads to duplicative efforts and wasted resources; and (5) providers frequently rely on patients to communicate about care taking place in the community.

CONCLUSIONS: VA providers described major challenges in coordinating with community providers, leading to perceptions of delayed, missed, or duplicative care and jeopardizing the overall quality, safety, and efficiency of Veteran care. Our study highlights the need for system-level solutions to support coordination across health systems for Veterans with COPD and may have implications for other conditions that lead to recurrent hospitalization and/or care in the community.

KEY WORDS: coordination; interdisciplinary communication; qualitative research; chronic obstructive pulmonary disease; electronic health record.

J Gen Intern Med 34(Suppl 1):S37-S42

DOI: $10.1007 / \mathrm{s} 11606-019-04971-2$

(C) Society of General Internal Medicine (This is a U.S. government work and not under copyright protection in the U.S.; foreign copyright protection may apply) 2019

Published online April 22, 2019

\section{INTRODUCTION}

High-quality healthcare depends on effective communication and coordination between healthcare providers. In recent years, there has been a rise in the number of providers comanaging patients with multimorbidity, resulting in increased care fragmentation. ${ }^{1}$ Fragmented care threatens healthcare quality and contributes to higher costs. ${ }^{2}$ In the absence of effective coordination, providers may have inadequate or inaccurate information, and patients may suffer from delayed, inappropriate, and/or erroneous care. ${ }^{3}$ Coordination is particularly important for patients who have multiple providers practicing in different health systems, as system-level barriers can impair the flow of patient information.

The Department of Veterans Affairs (VA) is the largest vertically organized healthcare system in the USA, supporting Veterans across the continuum of care and allowing diverse providers to communicate through established channels of a common electronic health record (EHR). Yet, Veterans are increasingly receiving care from "community providers," who we define as providers who are not employed by or contracted by the VA. This shift has been driven in part by implementation of the Veterans' Choice Act, which provides funds for Veterans with long wait times or travel distances to receive care from community providers. ${ }^{4}$ The Choice Act is being replaced by the VA MISSION Act of 2018, which is designed to consolidate VA community care programs and simplify the process by which Veterans receive community care. $^{5}$ This new legislation has the potential to further expand community care for Veterans, which highlights the need for effective coordination across health systems.

Veterans with chronic obstructive pulmonary disease (COPD) are particularly vulnerable to care coordination challenges. Several aspects of the disease process and patient population underscore the need for effective coordination. First, patients with COPD tend to have multimorbidity and multiple providers involved in their care. ${ }^{2,6,7}$ Second, they frequently receive community care which can impact clinical decisions by VA providers. ${ }^{8}$ Third, COPD is one of the most common causes of hospitalization, emphasizing the importance of coordination between inpatient and outpatient providers. ${ }^{9}$ Veterans who need emergent hospitalization may be admitted at the nearest community hospital, further complicating multi-system coordination. 
While several studies have explored outpatient provider perspectives on coordinating care between VA and community health systems, clear gaps in the literature exist. ${ }^{10-15}$ Most previous research focus on the experiences of community providers, who have expressed frustrations interacting with the VA system. ${ }^{10-12}$ Few studies have explored VA provider perspectives in this context, especially as they relate to coordinating across the continuum of care, including between inpatient and outpatient providers who practice in different health systems. Using COPD as an example, we sought to understand how VA providers perceive the status of multisystem coordination. Our goal was to inform and guide future cross-cutting initiatives to develop systems of communication and coordination between VA and community providers.

\section{METHODS}

\section{Study Design and Sample}

We conducted a qualitative study examining VA provider perspectives on collaborative care for Veterans with COPD from six geographically and organizationally diverse VA sites. We used purposive and snowball sampling methods to recruit providers who treat patients with COPD at the selected sites. ${ }^{16}$ To obtain a range of views on Veterans' care coordination, we intentionally enrolled some providers practicing primarily inpatient medicine and others practicing in outpatient settings. We included mental health providers in the study because COPD has high rates of mental health comorbidity (anxiety, depression, suicidal ideation). ${ }^{17}$ We emailed providers to explain the study and request their participation in the research, and we only included providers who responded to the request (opt-in approach). We continued interviewing participants until we reached thematic saturation, such that no new themes emerged with subsequent analysis. ${ }^{18}$ The project was approved by the Edith Nourse Rogers Memorial Veterans Hospital Institutional Review Board.

\section{Data Collection}

A researcher trained in qualitative methods (KR) conducted semi-structured telephone interviews at a time convenient for participants. The interview guide contained questions focused on communication about COPD care taking place in the community, including discharge information and clinic management. The interview guide was developed by the research team with input from two practicing pulmonologists (SR and $\mathrm{RW}$ ) and two qualitative researchers (KR and ARE). We focused on gaps in the literature relating to COPD management and transitions of care, especially regarding coordination across health systems. ${ }^{8}, 19$ Specific questions included (1) How do you collaborate with other providers to manage complex patients with multimorbidity, such as those with COPD?; (2) How do you communicate with non-VA providers?; (3) How do non-VA hospitals communicate discharge information to VA providers?; (4) What is the typical content of communication?; (5) What technologies are used to communicate?; (6) How are urgent patient issues (such as worsening condition) communicated?; and (7) What barriers to providing collaborative care for patients with COPD do you encounter?

We audio-recorded all interviews and contracted with a professional transcription service to transcribe recordings verbatim. After verifying transcriptions for accuracy, we uploaded the final transcription documents into NVivo 11 qualitative software for analysis. ${ }^{20}$

\section{Data Analysis}

Using principles of conventional content analysis, two researchers (SR and KR) performed a close reading and lineby-line analysis of transcripts to identify codes. ${ }^{21}$ We focused initial coding on the underlying research question: "How do VA providers perceive coordination with community providers for Veterans with COPD?" We (SR and KR) began by independently coding three transcripts, conventional content analytic methods, allowing for the emergence of inductive codes. ${ }^{18}$ When possible, we used phrases from the transcript to represent codes (e.g., "direct communication" to represent references to face-to-face or telephone conversations or "personal relationships" to represent statements that emphasize the importance of pre-existing relationships in facilitating coordination).

We compared coded transcripts between the two researchers, reviewing the code structure for logic, shared meanings, and comprehensiveness. We (SR and KR) met repeatedly to compare codes after each additional subset of five transcripts. In keeping with inductive coding methods, we continued to develop additional codes as new ideas emerged. We discussed the relevant sections of the transcripts to find common understanding of coded segments and discern and reconcile differences between coders. The entire research team met regularly during the analytic process to compare data, develop a codebook, and agree on emergent themes. We revised the codebook iteratively until we reached consensus among all team members. From these codes, we used inductive category development based on our research questions to generate themes that reflected commonalities that we encountered in interview responses. $^{22}$

\section{RESULTS}

We conducted interviews during the period of June to October, 2017. The final sample consisted of 25 providers, including 13 internal medicine providers, 10 pulmonologists, and two mental health providers (Table 1). The responding mental health providers had a particular interest in managing patients with COPD.

Qualitative data analysis yielded five themes: (1) VA providers perceive communication challenges between VA and 
Table 1 Characteristics of Interview Respondents by Site

\begin{tabular}{|c|c|c|c|c|c|c|c|c|c|}
\hline \multirow[t]{2}{*}{$\overline{\text { Site }}$} & \multirow[t]{2}{*}{ Region } & \multirow[t]{2}{*}{$n$} & \multicolumn{3}{|c|}{ No. of respondents by practice setting } & \multicolumn{3}{|c|}{ No. of respondents by specialty } & \multirow{2}{*}{$\begin{array}{l}\text { Average years } \\
\text { VA service }\end{array}$} \\
\hline & & & $\begin{array}{l}\text { Inpatient } \\
\text { only }\end{array}$ & $\begin{array}{l}\text { Outpatient } \\
\text { only }\end{array}$ & $\begin{array}{l}\text { Inpatient and } \\
\text { outpatient }\end{array}$ & $\begin{array}{l}\text { General } \\
\text { medicine }\end{array}$ & $\begin{array}{l}\text { Pulmonary } \\
\text { medicine }\end{array}$ & $\begin{array}{l}\text { Mental } \\
\text { health }\end{array}$ & \\
\hline A & West & 6 & 4 & 0 & 2 & 4 & 2 & 0 & 8 \\
\hline B & West & 5 & 1 & 1 & 3 & 3 & 2 & 0 & 12 \\
\hline $\mathrm{C}$ & Midwest & 5 & 1 & 3 & 1 & 3 & 1 & 1 & 9 \\
\hline E & Midwest & 3 & 1 & 1 & 1 & 2 & 1 & 0 & 8 \\
\hline D & South & 3 & 1 & 1 & 1 & 2 & 0 & 1 & 12 \\
\hline $\mathrm{F}$ & South & 3 & 1 & 2 & 0 & 3 & 0 & 0 & 5 \\
\hline
\end{tabular}

community providers, including difficult, inadequate, and delayed communication; (2) communication is facilitated by personal relationships across health systems; (3) the lack of electronic health record (EHR) interoperability impairs communication, resulting in transmission of unstructured data; (4) poor communication leads to duplicative efforts and wasted resources; and (5) providers frequently rely on patients to communicate about care taking place in the community.

Communication challenges. Respondents in our study perceived communication challenges with community providers, and frequently described this communication as "difficult," "insufficient," and "delayed." One respondent noted, "I think there's this sense of it's so hard so why should I try. I'm just not going to bother. It's too hard to get records...it's sort of this learned helplessness." Obtaining information on hospitalizations in the community was particularly challenging, as another provider noted, "Rarely, if ever, do we see things ahead of time or even contemporaneously. Not infrequently the type of information that comes is insufficient." Poor communication about hospitalizations in the community can place an added burden on VA providers as described by another respondent, "My perspective is [communication with community providers] doesn't happen a lot and that's often incumbent on the VA to go find the information once they learn that the patient was admitted."

Personal relationships. In our study, establishing or capitalizing on relationships with community providers was described as the best way to facilitate communication: "Unless the providers have a personal relationship, we never hear from the outside hospitals." Community providers who trained at a VA site were more likely to contact VA providers, as according to one provider, they have "preexisting knowledge and they're able to kind of break into the VA and find me." These preexisting relationships opened lines of communication that would have otherwise stayed closed.

Lack of EHR interoperability. Study participants repeatedly identified challenges with connectivity due to a lack of EHR interoperability. They acknowledged that interoperability is a pervasive challenge: "I think it's a frustration of anybody at any hospital in the United States because we don't have any centralized medical record." However, respondents described added layers of VA security that can impair communication: "[VA] has stunningly robust information security walls... sometimes it feels like we have stunningly well-protected information, [in a] medical information system that can't communicate." A lack of EHR interoperability results in transmission of unstructured data, such as letters and faxes that are subsequently scanned as PDF documents, posing barriers to providers in their ability to rapidly find, access, and search for pertinent clinical information.

Duplicative efforts and wasted resources. Poor coordination frequently resulted in unnecessary care and duplicated tests. This effect was summarized by one provider: "Sometimes [patients] come back to us with questions, concerns, or prescriptions, and then we would get involved and it's kind of a duplicative effort. We need to figure out what was done or what wasn't done and why they're asking for things." Imaging studies were a frequent source of duplicative efforts and wasted resources, as radiographs conducted outside VA were challenging to obtain and/or software was not always compatible. As one participant said, "it is almost easier to obtain another study at the VA... which is obviously a waste of resources."

Reliance on patients. Respondents in our study described relying on patients to act as intermediaries for communication with community providers: "Basically, our form of communication [with community providers] is periodically the patient bringing records..." Several respondents indicated that even hospitalizations were communicated through patients: "It's up to the patient to bring their discharge summary with them [to clinic]." When VA providers attempted to communicate with community providers, they still relied on patients to facilitate the communication. For example, one respondent indicated that they typically asked patients to provide contact information of their community providers.

\section{CONCLUSIONS}

VA providers identified major challenges in coordinating care with community providers, which may disproportionately 
affect Veterans with COPD or other complex chronic conditions as they navigate care from inpatient and outpatient providers across health systems. Qualitative data analysis identified key themes that related to the magnitude of coordination challenges, the causes and mitigators of coordination challenges (EHR interoperability and provider relationships), and the consequences of coordination challenges (reliance on patients and duplicated and wasted resources).

Providers in our study described difficult and delayed communication with other health systems, especially about hospitalizations that occurred in the community. Similar care coordination challenges were noted in a previous qualitative study of coordination outside the VA system between community PCPs and hospitalists, in which providers described the need to enhance interpersonal relationships, clearly define accountability, and promote information exchange through a shared EHR. ${ }^{23}$ While many of these themes were similar in our study, the recurrent hospitalizations among COPD patients and the unique features of VA, practice, may place additional stress on provider coordination. These repeated hospitalizations in the community could reinforce dysfunctional patterns of poor coordination. One provider in our study described developing a sense of "learned helplessness" about the ability to communicate with community providers, implying that some providers have capitulated to a standard of poor coordination. Overturning this perspective would likely require a transformative change in providers' roles and expectations. It is possible that even after barriers to coordination have been addressed, providers may need to be persuaded to proactively pursue multi-system coordination.

Conceptual frameworks for care coordination tend to present coordination as the task of exchanging information between members of a healthcare team, and few frameworks consider the unique challenges that exist for providers who coordinate care across health systems. ${ }^{24}$ The MacColl Institute's Care Coordination Model (CCM) presents coordination from the perspective of a patient-centered medical home (PCMH) and includes the importance of coordinating with external community agencies, hospitals, and specialists. ${ }^{25} \mathrm{PCMHs}$ have used this framework to guide policy and quality improvement efforts that promote coordination. $^{26-28}$ We found that many of the themes that emerged in our study reflected core constructs of the CCM, which emphasizes accountability, patient support, connectivity, and relationships and agreements between providers as critical components for delivering high-quality, coordinated care. ${ }^{25}$ Designated care coordinators may provide one evidence-based solution to reinforce these principles of coordination across health systems. For patients with COPD, care coordinators have been shown to reduce hospitalizations and improve quality of life. ${ }^{29}$ While care coordinators are commonly integrated into VA primary care, their responsibilities tend to focus on coordinating care within VA rather than communicating with community providers. ${ }^{30,}{ }^{31}$ Establishing designated care coordinators focused on supporting multi-system coordination could improve coordination for Veterans who receive care in the community.
Improving EHR interoperability can further support coordination by establishing lines of communication across health systems. Our results demonstrated that a lack of interoperability is a pervasive problem impairing coordination and leading to transmission of unstructured data. These findings reflect the core construct of connectivity in the CCM and are consistent with prior VA research. ${ }^{10-13}$ Legislation passed to support meaningful use of health information technology incentivizes health systems to use certified EHR technology that supports electronic information exchange. ${ }^{32}$ Despite this requirement, EHRs often fail to communicate important patient information in an effective and timely manner. ${ }^{33}$ Impaired interoperability has been particularly problematic for the VA, and ongoing efforts to upgrade the VA EHR are focused on improving the flow of information across health systems. ${ }^{34}$

Relationships between providers can further facilitate coordination and lead to agreements that specify coordinating roles and responsibilities. Providers in our study emphasized the importance of established relationships in coordinating care across health systems. The informal relationships that respondents described developed through personal contact and shared history, such as participating in medical training at the VA medical center. This emphasis on relationships is also highlighted in the CCM as essential to developing shared expectations for communication and care. In the absence of pre-existing relationships, contractual relationships or networks can develop through governance structures that promote coordination. ${ }^{35}$ To be effective, these structures need to engage diverse stakeholders, including front-line providers involved in patient care. ${ }^{36}$ Policies, regulations, and financial incentives can encourage contractual relationships and promote better coordination. ${ }^{37}$

The purpose of care coordination is to support patients as they receive recommendations from multiple providers who are often focused on different aspects of their care. Yet, providers in our study described frequently relying on patients to convey care that they receive in other health systems. This finding was also noted in a qualitative study of community providers, who described relying on Veterans as the main vehicle for information transfer between providers. ${ }^{12}$ Patients may not be willing or able to acquire their health records from community providers and share it with VA providers, or short of that, to verbally summarize health events, test results, and treatment changes in a timely and accurate fashion to ensure that their providers in different systems are on the same page. While this responsibility would be an undue burden on any sick patient or family member without a medical background, it is likely to be particularly onerous for the many Veterans with limited health literacy. ${ }^{38}$ Furthermore, direct communication between providers may be necessary to collaboratively manage patients with complex multimorbidity. ${ }^{39}$ It is therefore important and necessary that providers establish systems of coordination that do not depend on patient participation. Extending the principles of the PCMH model to support coordination across health systems could ensure that patients are supported and the need to rely on them as intermediaries is eliminated. 
This study has several strengths. We interviewed multiple providers practicing at geographically and organizationally diverse VA centers who were involved in diverse aspects of patient care, including inpatient and outpatient medicine. We used conventional content analysis, allowing inductive themes to emerge. After our inductive coding, we found that our results closely mirrored the existing framework in the CCM, which then served as a framework to generate potential solutions to address the challenges we found in coordinating care between VA and community providers. The research also has limitations. We were unable to observe coordinating practices and cannot determine whether provider perspectives reflected reality of clinical practice. Reassuringly, we heard common responses from multiple participants, and the themes that emerged from the data are well represented in the theoretical framework of the CCM.

Our study underscores the need for system-level solutions to support coordination for Veterans with COPD. It is likely that Veterans with other complex chronic conditions experience similar challenges with care coordination, especially for conditions that lead to recurrent hospitalization and/or care in the community. Implementation of the VA MISSION Act expands community care for many Veterans, which may augment the challenges of care coordination between VA and community providers and could threaten the quality of Veteran care. Providers and policymakers need to be aware of this threat and anticipate ways to counteract the deleterious effects of multi-system care. Adopting systems that incorporate principles of the Care Coordination Model could improve care for Veterans who receive treatment across health systems. Future efforts may include establishing care coordinators that support multi-system coordination, encouraging relationships and agreements across health systems, improving EHR interoperability, and focusing efforts on supporting patients in coordination efforts.

Corresponding Author: Seppo T. Rinne, MD PhD; , Bedford, MA, USA

\section{Compliance with Ethical Standards:}

The project was approved by the Edith Nourse Rogers Memorial Veterans Hospital Institutional Review Board.

Conflict of Interest: The authors declare that they do not have a conflict of interest.

Disclaimer: The views expressed in this article are those of the authors and do not necessarily represent the position or policy of the Department of Veterans Affairs or the US government.

\section{REFERENCES}

1. Pham HH, O'Malley AS, Bach PB, Saiontz-Martinez C, Schrag D Primary care physicians' links to other physicians through Medicare patients: the scope of care coordination. Ann Intern Med. 2009;150(4):236-242
2. Frandsen BR, Joynt KE, Rebitzer JB, Jha AK. Care fragmentation, quality, and costs among chronically ill patients. Am $J$ Manag Care. 2015;21(5):355-362.

3. Cebul RD, Rebitzer JB, Taylor LJ, Votruba ME. Organizational fragmentation and care quality in the U.S healthcare system. $J$ Econ Perspect. 2008;22(4):93-113.

4. Department of Veterans Affairs. Expanded Access to Non-VA Care Through the Veterans Choice Program. Federal Register. 2015;80(209):66419-66429.

5. The VA Mission Act of 2018. In: VA, ed.

6. Hussey PS, Schneider EC, Rudin RS, Fox DS, Lai J, Pollack CE. Continuity and the costs of care for chronic disease. JAMA Intern Med. 2014;174(5):742-748.

7. Han MK, Martinez CH, Au DH, et al. Meeting the challenge of COPD care delivery in the USA: a multiprovider perspective. Lancet Respir Med. 2016;4(6):473-526.

8. Rinne ST, Elwy AR, Bastian LA, Wong ES, Wiener RS, Liu CF. Impact of Multisystem Health Care on Readmission and Follow-up Among Veterans Hospitalized for Chronic Obstructive Pulmonary Disease. Med Care. 2017;55 Suppl 7 Suppl 1:S20-S25.

9. Wang L, Porter B, Maynard C, et al. Predicting risk of hospitalization or death among patients receiving primary care in the Veterans Health Administration. Medical care. 2013;51(4):368-373.

10. Pope CA, Davis BH, Wine L, et al. Perceptions of U.S. Veterans Affairs and community healthcare providers regarding cross-system care for heart failure. Chronic Illn. 2017;14(4):1742395317729887.

11. LaCoursiere Zucchero T, McDannold S, McInnes DK. "Walking in a maze": community providers' difficulties coordinating health care for homeless patients. BMC health services research. 2016;16:480.

12. Gaglioti A, Cozad A, Wittrock S, et al. Non-VA primary care providers' perspectives on comanagement for rural veterans. Mil Med. 2014;179(11):1236-1243.

13. Zuchowski JL, Chrystal JG, Hamilton AB, et al. Coordinating Care Across Health Care Systems for Veterans With Gynecologic Malignancies: A Qualitative Analysis. Medical care. 2017;55 Suppl 7 Suppl 1:S53-S60.

14. Tsai J, Yakovchenko V, Jones N, et al. "Where's My Choice?" An Examination of Veteran and Provider Experiences With Hepatitis C Treatment Through the Veteran Affairs Choice Program. Medical Care. 2017;55 Suppl 7 Suppl 1:S13-S19.

15. Mattocks KM, Mengeling $\mathbf{M}$, Sadler A, Baldor R, Bastian L. The Veterans Choice Act: A Qualitative Examination of Rapid Policy Implementation in the Department of Veterans Affairs. Medical care. 2017;55 Suppl 7 Suppl 1:S71-S75.

16. Valerio MA, Rodriguez $\mathbf{N}$, Winkler $\mathbf{P}$, et al. Comparing two sampling methods to engage hard-to-reach communities in research priority setting. BMC Med Res Methodol. 2016;16(1):146.

17. Panagioti M, Scott C, Blakemore A, Coventry PA. Overview of the prevalence, impact, and management of depression and anxiety in chronic obstructive pulmonary disease. Int $J$ Chron Obstruct Pulmon Dis. 2014;9:1289-1306.

18. Charmez K. Constructing Grounded Theory. Sage Publications Ltd; 2014.

19. Feemster LC, Au DH. Penalizing hospitals for chronic obstructive pulmonary disease readmissions. Am J Respir Crit Care Med. 2014;189(6):634-639.

20. NVivo qualitative data analysis Software [computer program]. Version 11: QSR International Pty Ltd; 2016.

21. Hsieh HF, Shannon SE. Three approaches to qualitative content analysis. Qual Health Res. 2005; 15(9):1277-1288.

22. Mayring P. Qualitative content analysis. Forum: Qualitative Social Research. 2000;1(2).

23. Jones CD, Vu MB, O'Donnell CM, et al. A failure to communicate: a qualitative exploration of care coordination between hospitalists and primary care providers around patient hospitalizations. J Gen Intern Med. 2015;30(4):417-424.

24. Van Houdt S, Heyrman J, Vanhaecht $\mathbf{K}$, Sermeus W, De Lepeleire J. An in-depth analysis of theoretical frameworks for the study of care coordination. Int $J$ Integr Care. 2013;13:e024.

25. Care Coordination Model. The MacColl Institute for Healthcare Innovation;2010.

26. Bodenheimer T, Ghorob A, Willard-Grace R, Grumbach $\mathbf{K}$. The 10 building blocks of high-performing primary care. Ann Fam Med. 2014;12(2):166-171.

27. Wagner EH, Sandhu N, Coleman K, Phillips KE, Sugarman JR. Improving care coordination in primary care. Med Care. 2014;52(11 Suppl 4):S33-38. 
28. Lee SJC, Jetelina KK, Marks E, et al. Care coordination for complex cancer survivors in an integrated safety-net system: a study protocol. BMC Cancer. 2018;18(1):1204.

29. Dajczman E, Robitaille C, Ernst P, et al. Integrated interdisciplinary care for patients with chronic obstructive pulmonary disease reduces emergency department visits, admissions and costs: a quality assurance study. Can Respir J. 2013;20(5):351-356.

30. Chang ET, Raja PV, Stockdale SE, et al. What are the key elements for implementing intensive primary care? A multisite Veterans Health Administration case study. Healthc (Amst). 2017.

31. Ayele RA, Lawrence E, McCreight $\mathbf{M}$, et al. Study protocol: improving the transition of care from a non-network hospital back to the patient's medical home. BMC health services research. 2017;17(1):123.

32. Centers for Medicare and Medicaid Services, Medicare and Medicaid Programs, and Electroic Health Record Incentive Program: Final Rule. In. Vol 75. Federal Register 2010:44314-44358.

33. Richardson JE, Vest JR, Green CM, Kern LM, Kaushal R, Investigators $\mathrm{H}$ A needs assessment of health information technology for improving care coordination in three leading patient-centered medical homes. $J$ Am Med Inform Assoc. 2015;22(4):815-820.
34. VA, Cerner Announce Agreement to Provide Seamless Care for Veterans [press release]. Cerner Corporation, May 17, 20182018.

35. Hawkins MA. Clinical integration across multiple hospitals: the agony, the ecstasy. Adv Pract Nurs Q. 1998;4(1):16-26.

36. Rogers A, Sheaff $\mathbf{R}$. Formal and informal systems of primary healthcare in an integrated system: evidence from the United Kingdom. Healthc Pap. 2000; 1(2):47-58; discussion 104-107.

37. Friedman L, Goes J. Why integrated health networks have failed. Front Health Serv Manage. 2001;17(4):3-28.

38. Chew LD, Griffin JM, Partin MR, et al. Validation of screening questions for limited health literacy in a large VA outpatient population. J Gen Intern Med. 2008;23(5):561-566.

39. Wallace E, Salisbury C, Guthrie B, Lewis C, Fahey T, Smith SM. Managing patients with multimorbidity in primary care. $B M J$. 2015;350:h176.

Publisher's Note Springer Nature remains neutral with regard to jurisdictional claims in published maps and institutional affiliations. 